\title{
The CARM beam-wave interaction and cavity design
}

\author{
E. Di Palma ${ }^{1}$, G. Dattoli ${ }^{1}$, S. Ceccuzzi ${ }^{1}$, E. Sabia ${ }^{1}$, G. L. Ravera ${ }^{1}$, I. Spassovsky ${ }^{1}$, F. Mirizzi ${ }^{2}$, A. Doria ${ }^{1}$, \\ A.A. Tuccillo ${ }^{1}$, G.P. Gallerano ${ }^{1}$ and the ENEA CARM Task Force \\ ${ }^{1}$ ENEA Fusion Department, C.R. Frascati, Via E. Fermi 45, 00044 Frascati, Roma, Italy \\ ${ }^{2}$ Consorzio CREATE, Via Claudio 21, 80125, Napoli, Italy
}

\section{Introduction}

A major objective to be pursued in the design of future fusion reactors is the decrease of re-circulated power, which draws a special attention to the wall-plug efficiency of heating and current drive systems. The conceptual study for a self-sustainable DEMO-nstration reactor foresees for the current drive systems an efficiency larger than $30 \%$ [1]. This represents a critical requirement to develop the future electron cyclotron systems for nextgeneration fusion devices.

Furthermore, a microwave tube satisfying DEMO requirements, i.e. able to provide large continuous-wave power $(\sim 1 \mathrm{MW})$ at high frequency $(\sim 250 \mathrm{GHz})$, needs intensive R\&D. Nowadays, the scientific community is devoting a special effort to extend above $200 \mathrm{GHz}$ the operational frequency range of the gyrotron, the most mature technology in the field of mm-wave tubes.

In this frame a research and development program has been undertaken at ENEA Frascati Research Center, aimed at realizing a microwave tube based on a Cyclotron Auto-Resonance Maser (CARM) oscillator [2], characterized by a high value of the frequency Doppler up-shift allowing a consistent reduction of the static magnetic field in the interaction cavity. The price to be paid is the necessity of exploiting high quality electron beams, with velocity spread below $0.5 \%$, in order to ensure appropriate mode selection, adequate wave-beam power transfer with the required efficiency. The design of the CARM cavity, composed by a short smooth cylindrical waveguide section delimited by two Bragg reflectors, is a challenging task, aimed at realizing the conditions to select the right operational mode and to limit the growth of the competing modes.

The radio frequency circuit design must be supported by a numerical model allowing to accurately simulate the intra-cavity beam wave interaction. The large length of this cavity $(\sim 80 \mathrm{~cm})$ compared to the wavelength under investigation impairs the effectiveness of a classical particle in cell code (PIC), based on the discretization of the Maxwell equations, due to the huge amount of computer memory and CPU time required by the resulting mesh sizes.

In parallel to the development of the full numerical analysis, we have undertaken the cavity design by the use of "home-made" 1D/3D code, based on the selfconsistent procedure discussed by several authors [3-5] and on the "universal" scaling formulae that describe the CARM performances including inhomogeneous broadening effects and the beam current, as it happens in the case of Undulator FEL [6]. An effective analysis tool in term of analytical formulae and computer simulation has been accordingly obtained.
The benchmarking of both the numerical code and semi-analytical formula with a test case (coming from an experimental CARM amplifier test facility developed at the Massachusetts Institute of Technology) at low frequency $(\sim 35 \mathrm{GHz})$ reported in ref. [5] has been discussed in ref. [7].

The agreement between the procedure, we have envisaged, and the experimental results, has provided some confidence on the reliability of the method. Therefore the application of this procedure to the preliminary design of the ENEA 250-GHz CARM resonant cavity is presented. The analysis allows the understanding of the conditions to be fulfilled by the cavity Q-factor, by the length of the smooth cylindrical waveguide section and by the beam parameters, in order to achieve a convenient compromise among the CARM required efficiency and a relatively low value of the starting current.

\section{Results}

The beam-wave interaction in a cold cavity for a CARM oscillator opens three kinds of problems as highlighted in Fig. 1:

a) the suppression of the beam-wave intersection for the operating mode at low frequency;

b) the suppression of the excitation of the competitor mode near cut-off;

c) the suppression of the modes competition near the Doppler up-shift interaction.

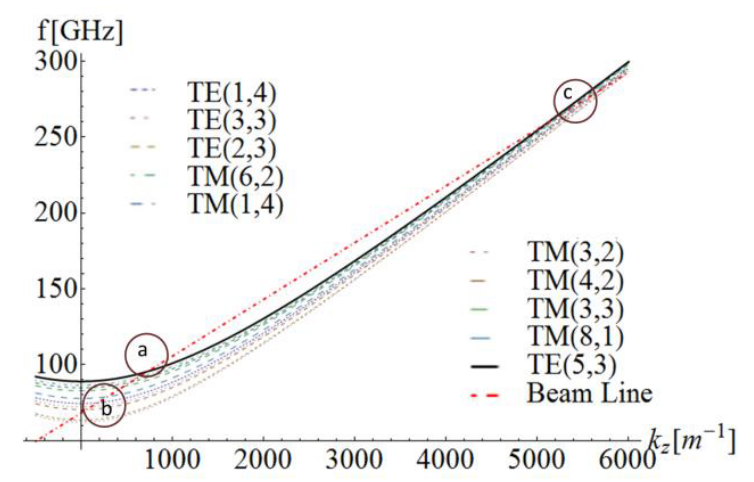

Fig. 1. Brillouin diagram of a particle beam $\left(\gamma=2.17, \mathrm{v}_{\perp} / \mathrm{v}_{\|}=\right.$ $=0.53$ ) interaction with modes of a cylindrical cold cavity with radius $0.75 \mathrm{~cm}$

The solution for the point a), widely discussed in ref. [7], is given by adjusting the design parameters in such a way that the starting current [4] of the near cutoff mode (intersection point a in Fig. 1) is greatly enhanced with respect to the CARM interaction. The underlying optimization is sketched in the block diagram in Fig. 2. The search for an optimum interaction length is based on a 
compromise between gain and efficiency for a given output CARM oscillator power.

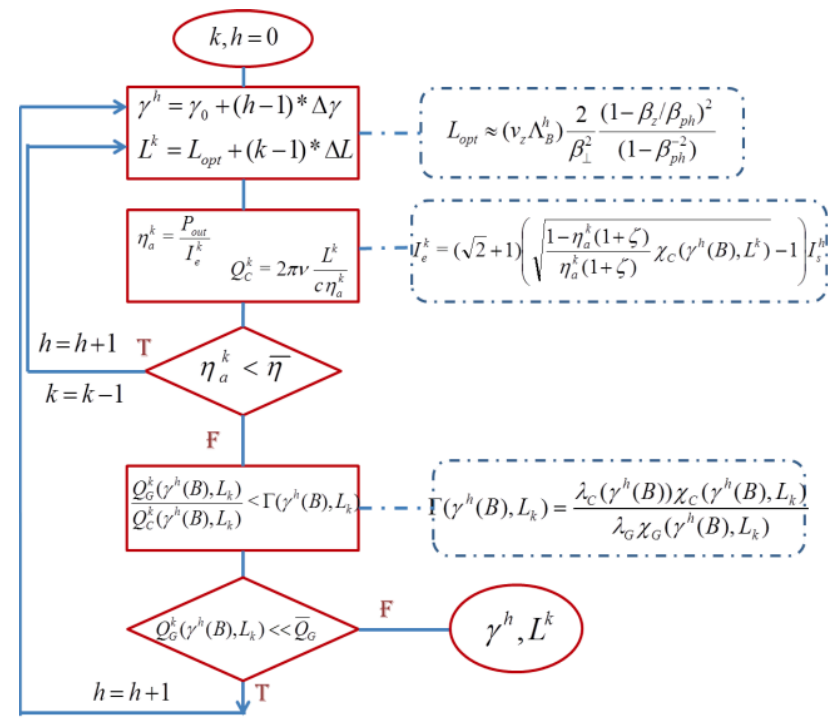

Fig. 2. Block diagram describing the procedure allowing to fix the beam energy and the smooth cylindrical cavity section length limiting the growing of the lower beam intersection with the operating mode

Major attention must be paid to the point b) for which the increase of the starting current is not sufficient for suppressing the modes growth excited by the beam near the cut-off where TM modes play a crucial role too.

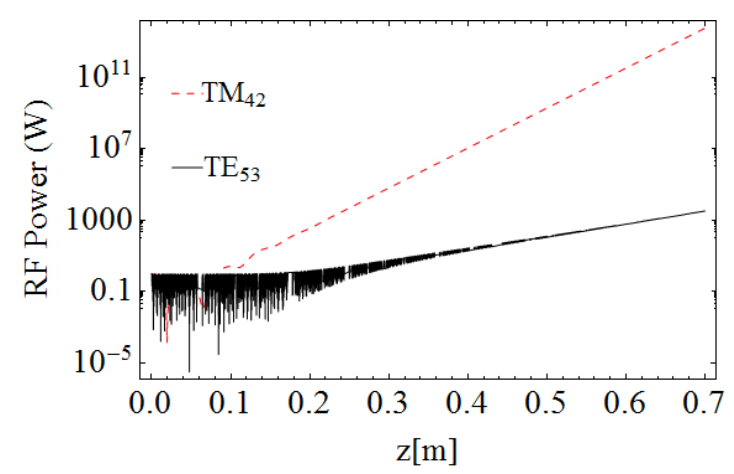

Fig. 3. The linear growth signal for an electron beam (with $\gamma=2.17$, $\mathrm{v}_{\perp} / \mathrm{v}_{\|}=0.53, \mathrm{I}=8 \mathrm{~A}$ ) interacting with the modes of a cylindrical cavity of radius $\mathrm{R}=7.5 \mathrm{~cm}$

In Fig. 3 it has been put in evidence how large is the linear growth signal for the $\mathrm{TM}_{42}$ at $70 \mathrm{GHz}$ (near cutoff) with respect to the operating mode $\mathrm{TE}_{53}$ at $259 \mathrm{GHz}$. The starting current for TM modes is minimized at the cut-off, since the group velocity of the wave is well separated from the particle velocity, thus increasing the beamwave energy exchange. Opposite condition occurs at CARM resonance implying an infinity value for the starting current. Due to the low value of the starting current and the high growth factor, the only way to suppress the TM mode is by using of a slotted cavity, suppressing the mode rotation, but causing a reduction of $30 \%$ of the system overall efficiency. The mode competition at CARM interaction (see point $\mathrm{c}$ in Fig. 1) can be kept under control by operating with an electron beam with a moderate velocity spread (lower than $0.5 \%$ ).
This statement is easily understood, by noting that the dependence of the CARM frequency on the longitudinal particle beam velocity $v_{z}$ (for fixed $\gamma$ beam energy) can be derived by coupling the dispersion relation of the beam-wave interaction with that of the propagating mode in a cylindrical cavity, which yields

$$
\omega=\frac{-\beta_{z}^{-2} \Omega / \gamma-\sqrt{\omega_{\text {cutoff }}^{2}\left(1-\beta_{z}^{-2}\right)+\beta_{z}^{-2} \Omega^{2} / \gamma^{2}}}{1-\beta_{z}^{-2}}
$$

being $\Omega$ the cyclotron frequency determined by the axial magnetic field, $\omega_{\text {cutoff }}$ the cut-off frequency of the propagating mode and $\beta_{z}=v_{z} / c$ the normalized longitudinal velocity of the particle beam. In Fig.4 we have plotted Eq. 1 vs. $v_{\mathrm{z}}$ for a beam with $\gamma=2.17$ and $\alpha=\mathrm{v}_{\perp} / \mathrm{v}_{\|}=0.53$ interacting with the modes supported by a cylindrical cavity of radius $7.5 \mathrm{~mm}$. The plot shows that, for an electron beam within the quoted r.m.s velocity limits, the simultaneous locking of different modes does not occur. A thoroughly discussion of the ENEA CARM oscillator design criteria, along the previously sketched lines, will therefore follow.

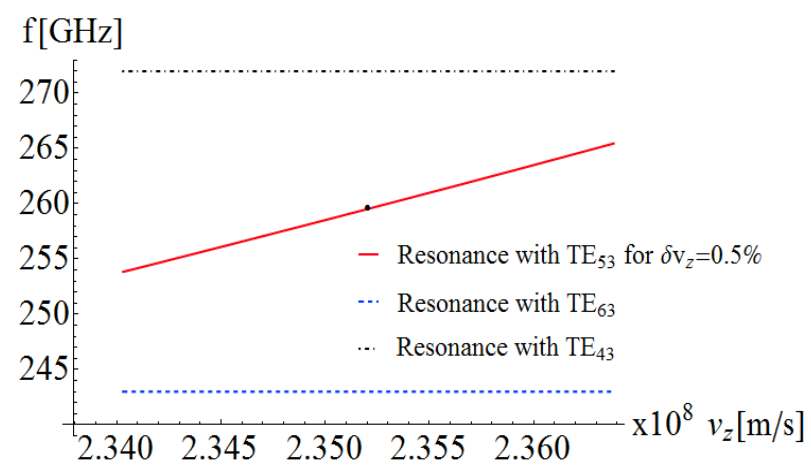

Fig. 4. The resonance frequency range for a beam line with an energy spread $(0.5 \%)$ interacting with the $\mathrm{TE}_{53}$ mode, well separated from the resonance with the competitors modes $\mathrm{TE}_{63}$ (dotted line) and $\mathrm{TE}_{43}$ (dot-dashed line)

\section{References}

1. Zhom,H. On the minimum size of DEMO // Fusion Sci. Technol. 2010, V. 58 P. 613-624.

2. Artioli M. et al., on behalf of CARM TEAM, A $250 \mathrm{GHz}$ Radio Frequency CARM Source for Plasma Fusion, Conceptual Design Report // edited by ENEA, online at: http://www.enea.it/ it/pubblicazioni/edizionienea/2016/conceptual-design-report (ISBN: 978-88-8286-339-5)

3. Petelin, M.I. On the theory of ultrarelativistic cyclotron self-resonance masers // Izvestiya VUZ. Radiofizika 1974, V. 17(6) P. 902-908.

4. Bratman, V.L., Ginzburg, N.S., Nusinovich, G.S. Petelin, M.I. and Strelkov, P.S. Relativistic gyrotrons and cyclotron autoresonance maser // Int. J. Electronics 1981, V. 51(4) P. 541567.

5. Chen, C. and Wurtele, J.S. Linear and nonlinear theory of cyclotron autoresonance masers with multiple waveguide modes // Phys. Fluids B 1991, V. 3(8) P. 2133-2148.

6. Dattoli, G., Ottaviani, P.L., Pagnutti, S. Booklet for FEL design: a collection of pratical Formulae // edited by ENEAEdizioni scientifiche, October 2007.

7. Di Palma, E., Dattoli, G., Sabia, E., Sabchevski, S., Spassovsky, I. Beam-wave interaction from FEL to CARM and associated scaling laws, to be submitted. 